\title{
Mapping the Milky Way with LSST
}

\author{
Željko Ivezić (for the LSST Collaboration) \\ Department of Astronomy, University of Washington, Seattle, WA 98155, USA \\ email: ivezic@astro.washington.edu
}

\begin{abstract}
The Large Synoptic Survey Telescope (LSST) is the most ambitious ground-based survey currently planned in the visible band. Mapping of the Milky Way is one of the four main science and design drivers. The main 20,000 sq.deg. large survey area will be imaged about 1000 times in six bands (ugrizy) during the anticipated 10 years of operations, with the first light expected in 2015. These data will result in databases including 10 billion galaxies and a similar number of stars, and will serve the majority of science programs. In the Milky Way context, these deep LSST data will enable unprecedented studies of distant stars in Galactic halo, as well as intrinsically faint nearby objects, such as white dwarfs and LTY dwarfs.
\end{abstract}

Keywords. Surveys, atlases, catalogs, astronomical data bases, astrometry, photometry

LSST will be a large, wide-field ground-based system designed to obtain multiple images covering the sky that is visible from Cerro Pachón in Northern Chile. The LSST design is driven by four main science themes: constraining dark energy and dark matter, taking an inventory of the Solar System, exploring the transient optical sky, and mapping the Milky Way. The current baseline design, with an $8.4 \mathrm{~m}(6.7 \mathrm{~m}$ effective) primary mirror, a $9.6 \mathrm{deg}^{2}$ field of view, and a 3,200 Megapixel camera, will allow about 10,000 square degrees of sky to be covered using pairs of 15-second exposures in two photometric bands every three nights on average. The system is designed to yield high image quality as well as superb astrometric and photometric accuracy. The survey area will include 30,000 $\operatorname{deg}^{2}$ with $\delta<+34.5^{\circ}$, and will be imaged multiple times in six bands, ugrizy, covering the wavelength range $320-1050 \mathrm{~nm}$. About $90 \%$ of the observing time will be devoted to a deep-wide-fast survey mode which will observe a 20,000 $\mathrm{deg}^{2}$ region about 1000 times in the six bands during the anticipated 10 years of operations. These data will result in databases including 10 billion galaxies and a similar number of stars, and will serve the majority of science programs. The remaining $10 \%$ of the observing time will be allocated to special programs such as Very Deep and Very Fast time domain surveys. More information about LSST can be obtained from www.lsst.org and Ivezić et al. (2008).

As a comparison, each 30-sec observation will be about 2 mag deeper than SDSS imaging, and the repeated observations will enable proper motion and trigonometric parallax measurements to $r=24.5$, about 4-5 mag fainter limit than to be delivered by Gaia, and the coadded LSST map will reach $r=27.5$. Due to Gaia's superb astrometric and photometric accuracy, and LSST's significantly deeper data, the two surveys are highly complementary: Gaia will map the Milky Way's disk with unprecedented detail, and LSST will extend this map all the way to the halo edge.

\section{References}

Ivezić, Ž., Tyson, J. A., Allsman, R. et al. 2008, arXiv:0805.2366 